\title{
Lane-Based Short-Term Urban Traffic Parameters Forecasting Using Multivariate Artificial Neural Network and Locally Weighted Regression Models: A Genetic Approach
}

\begin{tabular}{|c|c|}
\hline Journal: & Canadian Journal of Civil Engineering \\
\hline Manuscript ID & cjce-2017-0644.R1 \\
\hline Manuscript Type: & Article \\
\hline $\begin{array}{l}\text { Date Submitted by the } \\
\text { Author: }\end{array}$ & 18-Apr-2018 \\
\hline Complete List of Authors: & $\begin{array}{l}\text { Raza, Asif; Wuhan University of Technology, Intelligent Transportation } \\
\text { Research Center } \\
\text { Zhong, Ming; Wuhan University of Science and Technology, Civil } \\
\text { Engineering }\end{array}$ \\
\hline Keyword: & $\begin{array}{l}\text { Short-term forecasting, Genetic algorithms, Artificial neural network, } \\
\text { Locally weighted regression }\end{array}$ \\
\hline $\begin{array}{r}\text { Is the invited manuscript for } \\
\text { consideration in a Special } \\
\text { Issue? }\end{array}$ & Transportation Safety under Big Data \\
\hline
\end{tabular}




\section{Lane-Based Short-Term Urban Traffic Parameters Forecasting Using Multivariate}

\section{2}

Artificial Neural Network and Locally Weighted Regression Models: A Genetic Approach

\section{Asif Raza ${ }^{1}$, Ming Zhong ${ }^{2}$}

${ }^{1}$ National Engineering Research Center for Water Transport Safety; Engineering Research Center

7 for Transportation Safety, China Ministry of Education; Intelligent Transportation Research

8 Center, Wuhan University of Technology, Wuhan, 430063, Hubei, P.R. China.

9 Tel: +86-18717133530; Email raza@whut.edu.cn

$11 \quad{ }^{2}$ National Engineering Research Center for Water Transport Safety;

12 Intelligent Transport Systems Research Center, Wuhan University of Technology;

13 Adjunct Professor, Dept. of Civil and Environmental Engineering, University of Waterloo;

14 P.O.Box 125, 1040 Heping Avenue, Wuhan, 430063, P.R. China.

15 Tel: +86-(27) 86582280; Email: mzhong@whut.edu.cn

18 Word Count: 8969 words [6969 words for text (abstract, main text, and references); 2000 words

1920 for tables and figures (3 table and 5 figures)] 


\section{$21 \quad$ Abstract}

22 Short-term prediction of traffic conditions on urban arterials has recently become increasingly

23 important because of its vital role in the basic traffic management functions and trip decision-

24 making processes. Such information is useful for optimal infrastructure operation, routing and trip

25 scheduling. However, forecasting models offering a high accuracy at a fine temporal resolution

26 (e.g., 1 or $5 \mathrm{~min}$ ) and, especially, lane-based are still rare and need special attention. Given the

27 dynamic and stochastic nature of traffic, this study proposes a genetically optimized artificial

28 neural network (GA-ANN) and locally weighted regression (GA-LWR) multivariate models, for

29 short-term traffic prediction using a combination of multiple traffic variables such as volume,

30 occupancy and speed, during peak and off-peak periods. The proposed 5-min GA-ANN and GA-

31 LWR disaggregate multivariate models show lower average and the 95th percentile (P95) errors,

32 when compared to those reported in the literature. In particular, for peak and off-peak time

33 prediction, the GA-ANN disaggregate multivariate models result in most of average errors of 2-

$345 \%$ and the 95 th percentile errors of $9-10 \%$. On the other hand, for peak and off-peak time traffic

35 prediction, the GA-LWR disaggregate multivariate models show most of the average errors lower

36 than $5 \%$ and of $95^{\text {th }}$ percentile errors are lower than $10 \%$. Meanwhile, for peak and off-peak time

37 prediction, both GA-ANN and GA-LWR disaggregates models show lower MSE of 0.11-1.84.

38 Hence, such techniques are believed useful for developing a robust urban traffic forecasting 39 system.

40

41 Keywords: Short-term forecasting, Genetic algorithms, Artificial neural network, Locally weighted regression, Aggregate model, Disaggregate model. 


\section{1. Introduction}

Intelligent transportation systems (ITS), in recent years, have achieved enormous

46 developments. ITS applications improve the overall efficiency of transportation systems and

47 mitigate transportation related issues using information and communication technologies.

48 Increasing traffic demand requires an efficient and proper operation of transportation

49 infrastructure. Literature on short-term traffic flow forecasting has undergone a significant

50 development. It has been noticed from the literature that traffic forecasting is a crucial task in the

51 area of ITS. During the peak hours, when the amount of traffic demand exceeds the available

52 capacity of the road network, congestion occurs and it creates bottlenecks in urban areas. Traffic

53 forecasting, especially short-term traffic flow forecasting is momentous to modern ITS. It is the

54 process of estimating anticipated traffic conditions using historical traffic information and

55 intelligent controls. The history of short-term traffic forecasting is long and many researchers

56 (Vlahogianni et al. 2014; Smith \& Demetsky 1994) have made numerous contributions in many

57 ways given its diversity in nature.

The lane-based analysis explores traffic characteristics of each lane separately to analyse

59 different traffic patterns during peak and off-peak time of the day. Lane-based routing is a valuable

60 strategy for reducing serious traffic delays during the peak traffic period. The available

61 infrastructure is limited in terms of the capacity, so it is important to utilize the available capacity

62 in an optimal way, hence lane-based routing system will not only enhance the available network

63 capacity but also help mitigate traffic jams. It contributes to enhancing traffic operation and

64 implementing better traffic control and signal system.

65 Literature shows that most of the previous studies have focused on rural and highway 
66 traffic predictions and most of them are direction-based. Although various methodologies have

67 been proposed and applied for, forecasting short-term traffic, and the ultimate goal remains the 68 same: to obtain the forecasting results as precisely and quickly as possible. Researchers are

69 improving and optimizing the existing methodologies to develop new models in this area.

It has been noticed from previous studies that genetic algorithms are one of the best optimization techniques available to optimize complex and non-linear dynamic behavior of traffic.

72 In this study, two types of models are proposed and developed. 1) the aggregate multivariate 73 model: this model forecasts traffic data of six-intervals of 5-min window each weekday. 2) the 74 disaggregate multivariate model: this model only forecasts one-interval of 5-min window each 75 weekday. Both models are used to predict short-term traffic pattern using multiple traffic variables 76 during peak and off-peak period.

Given the heterogeneous nature of urban traffic, inputs consisting of a combination of multiple traffic variables will play a vital role in developing a robust model, which will help predict current traffic conditions using rich historical data. Hence, short-term lane-based forecasting using multivariate models could offer a higher accuracy at a fine temporal resolution (e.g., 1 or 5 min).

This paper is divided into separate sections as follows. The next section of this paper 82 gives a brief review of inclusive research in the area of short-term traffic forecasting. The third 83 section presents details about the optimization and modelling techniques used. The fourth section 84 presents the details about study data and initial processing. The fifth section provides relevant 85 details about the study models. The sixth section describes the implementation of GA designed 86 ANN and LWR aggregate and disaggregate multivariate models. The seventh section gives 87 detailed discussion about models and results. Last, the paper will present the principal findings, 
88 relevant conclusions, and recommendations of this study.

\section{A Review of Short-Term Traffic Forecasting}

90

ITS, in recent years have showed great progress. The main aim of such systems is to

91 improve the current traffic conditions using advanced information and communication tools.

92 Traffic congestion is one of the major concern of developed and developing countries. To

93 overcome the rapidly increasing traffic problems, it is important to introduce such models, which

94 will enhance the traffic prediction accuracy (Khan \& Maini 2000).

95 Short-term traffic forecasting is an important component of today's modern ITS.

96 Researchers have focused on developing short-term traffic forecasting methodologies that can be

97 used to estimate near-term traffic characteristics such as traffic flow, speed and occupancy

98 (Vlahogianni et al. 2014). Accuracy of short-term traffic forecasting algorithms extensively

99 depends on the system's availability and methods used. It is essential to understand the working

100 process behind all the methods to get an idea of the limitations associated with each of them.

101 Numerous methodologies have been applied to short-term traffic forecasting depending on data

102 types. The objectives are to develop such a system, which is used to increase the operational

103 efficiency and capacity of the transportation network. Many methods and procedures are adopted

104 to predict different traffic parameters, such as time series analysis, real-time methods, historical

105 methods, statistical methods and machine learning methods (Kumar et al. 2013).

The work of Box and Jenkins 1970 opened a gateway to time-series methods for short-term

107 traffic prediction. In the early 90's, autoregressive integrated moving average (ARIMA), family

108 of models were extensively used for traffic forecasting (Hamed et al. 1995; Kirby et al. 1997; 
The forecasts produced using seasonal ARIMA (SARIMA) model produced more accurate

111 results than non-seasonal ARIMA models as SARIMA models dealing better with the seasonality

112 of the traffic and showed average errors of $7.41 \%$ (Williams et al. 1998). The non-parametric

113 regression approaches show better accuracy than traditional ARIMA models and Kalman filtering

114 models (Smith \& Demetsky 1997; Smith et al. 2002; Okutani \& Stephanedes 1984). The ARIMA

115 models and other time-series methods are linear by nature. However, traffic patterns are nonlinear

116 and random by nature. Hence, these methods cannot fully address the traffic dynamics and 117 therefore the prediction errors are generally high.

Artificial neural networks (ANNs) are among the most widely used alternatives for

119 modelling heterogeneous traffic conditions, since they can approximate almost any function,

120 regardless of its degree of nonlinearity and without prior knowledge of its functional form

121 (Vlahogianni et al. 2005). As per extant literature, academicians have widely used ANN models

122 to predict short-term traffic conditions (Centiner et al. 2010; Hu et al. 2010; Pamula 2011;

123 Dougherty \& Cobbett 1997). ANN urban traffic modelling and prediction results based on

124 simulation showed higher accuracy, especially for short-term traffic flow (Ledoux 1997). ANN

125 models were used to predict short-term traffic volume and results showed that such learning 126 produced better results (Yasdi 1999). ANN was used to predict traffic parameters using historical 127 traffic data to predict short-term traffic and results showed that artificial neural network performed 128 well for traffic flow prediction, even when the time window increased from 5-min to 30-min (4). 
131 volumes (Lingras \& Adamo 1996). A nonparametric dynamic time-delay recurrent wavelet neural 132 network model was used to forecast traffic flow (Jiang \& Adeli 2005) and the developed models 133 produced satisfactory results when applied to long-term and short-term traffic flow forecasting. A 134 combination of genetic algorithm and neural network was used for short-term traffic flow 135 prediction and the developed MPL network performed well and produced satisfactory results on 136 both univariate and multivariate traffic flow data types, acquired from an urban signalized arterial 137 road (Vlahogianni et al. 2005). A fuzzy neural network known as the pseudo outer-product fuzzy 138 neural network was used for short-term traffic flow prediction and the developed models produced 139 higher accuracy as compared to traditional feed-forward neural networks (Quek et al. 2006). Two 140 non-parametric models, non-parametric regression (NPR) and the Gaussian maximum likelihood 141 (GML) was used for daily traffic flow forecasting based on historical data type collected for the 142 annual traffic census (ATC) in Hong Kong and results showed that these models produced better 143 results; especially the NPR model forecasts produced better results than the GML model (Lam et 144 al. 2006). A nearest neighbour form of nonparametric regression, coupled with a monitoring 145 system was used to monitor the current traffic conditions and verify the results, based on historical 146 data to evaluate the traffic flow deviation from the past traffic flow (Turochy 2006). Two Wavelet 147 networks were used to predict short-term traffic flow and the developed models showed better 148 results compared with the well-known back-propagation neural network (BPNN) and radial basis 149 function neural network (RBFNN) models. The results showed that the developed models 150 produced lower errors than the BPNN and RBFNN models (Xie \& Zhang 2006).

152 regression (LSVR) for short-term traffic flow forecasting (Theja \& Vanajakskshi 2010; Liu et al. 153 2007). It has been noticed from literature that there are a handful studies related to genetically 
154 designed hybrid models for traffic flow (hourly-based) forecasting. The genetically designed 155 neural network and regression models, factor models, and ARIMA models were used to estimate 156 missing traffic counts and the designed models produced lower average and the 95th percentile 157 errors, especially for refine models with stable counts. Meanwhile, even unstable traffic patterns 158 produced lower average and the 95th percentile errors (Zhong et al. 2004). A hybrid genetically 159 optimized regression and time delay neural network models were used to predict hourly-based 160 traffic volumes for different types of road and the developed the refined regression models showed 161 lower average and 95th percentile errors as compared to time delay neural network models (Zhong 162 et al. 2005). Hybrid models have received much attention because of their capability to model 163 heterogeneous traffic flow conditions and results are more accurate and more promising, than 164 standalone approaches (Zeng et al. 2008). A hybrid wavelet transform (WT) and support vector 165 machine (SVM) model were used for short-term traffic flow prediction (Yonghui et al. 2011). 166 Meanwhile, a hybrid Chaos-Wavelet Analysis-Support Vector Machine (C-WSVM) was used for 167 short-term real traffic speed data forecasting and found that the developed model produced high 168 satisfactory results (Wang \& Shi 2013). A hybrid approach integrating the fuzzy C-means (FCM)169 based imputation method with genetic algorithm was used to estimate missing traffic volume data 170 and the developed model performed well compared to conventional Exponential Smoothing, and 171 ARIMA methods and showed average RMSE error of 42.84\% (Tang et al. 2015). An absorbing 172 Markov chain (AMC) model was developed to predict short-term traffic volume using historical 173 time series data, which significantly outperformed SARIMA and neural network models (Mei et 174 al. 2015). Locally weighted regression (LWR) is a memory-based nonparametric approach and it 176 mapped the real-valued input vectors to the real-valued output vectors. Local modelling can learn 
177 locally as opposed to global models like neural network and it is used to predict the future traffic

178 patterns using the past available traffic data (Friedman 1995; Cleveland \& Devlin 1988). The

179 memory-based methods are often favoured over other global methods, when dealing with non-

180 stationary input distribution. It uses spatially localized algorithms, such as nearest neighbor

181 methods, kernel regression and lazy learning (Atkeson et al. 1997) and it has found that local linear

182 methods are more accurate than other methods such as k-nearest and the kernel smoothing

183 methods. Local modelling is preferred other than global modelling like neural network because

184 such models not only offer a higher accuracy but also they can avoid negative interference

185 generated by global models (Atkeson et al. 1997b). The LWR learning systems grow as it tries to

186 compute the model and these models are comparatively inexpensive to compute and used to

187 forecast short-term traffic flow (Sun et al. 2003).

189 Table 1 short Summary of previous studies

\begin{tabular}{|c|c|c|c|c|c|}
\hline $\begin{array}{l}\text { Year } \\
\text { of } \\
\text { the } \\
\text { study }\end{array}$ & Authors & $\begin{array}{l}\text { Data used/Data } \\
\text { Type }\end{array}$ & $\begin{array}{l}\text { Forecasting } \\
\text { techniques used }\end{array}$ & Accuracy & Note \\
\hline \multirow[t]{2}{*}{1998} & \multirow[t]{2}{*}{$\begin{array}{l}\text { Williams et } \\
\text { al. }\end{array}$} & \multirow[t]{2}{*}{ hourly traffic flows } & \multirow{2}{*}{$\begin{array}{l}\text { Statistical models for } \\
\text { forecasting on two } \\
\text { urban freeways }\end{array}$} & $\begin{array}{l}\text { Average seasonal } \\
\text { ARIMA }=7.41 \%\end{array}$ & $\begin{array}{l}\text { ARIMA = autoregressive integrated } \\
\text { moving average }\end{array}$ \\
\hline & & & & ESM $7.49 \%$ & ESM $=$ Winters exponential smoothing \\
\hline \multirow[t]{4}{*}{2005} & \multirow[t]{4}{*}{$\begin{array}{l}\text { Vlahogianni } \\
\text { et al. }\end{array}$} & \multirow[t]{4}{*}{ Traffic flow } & \multirow{4}{*}{$\begin{array}{l}\text { Genetically designed } \\
\text { optimized and meta- } \\
\text { optimized neural } \\
\text { network }\end{array}$} & UGDMAE $=14 \%$ & $\begin{array}{l}\text { UGDMAE }=\text { Univariate Gradient } \\
\text { Descent Mean Absolute Error }\end{array}$ \\
\hline & & & & $\begin{array}{l}\text { UGDMAPE } \\
=21.71 \% .\end{array}$ & $\begin{array}{l}\text { UGDMAPE =Multivariate Gradient } \\
\text { Descent Mean Absolute Percentage } \\
\text { Error }\end{array}$ \\
\hline & & & & MGDMAE $=13 \%$ & $\begin{array}{l}\text { MGDMAE }=\text { Multi Gradient Descent } \\
\text { Mean Absolute Error }\end{array}$ \\
\hline & & & & $\begin{array}{l}\text { MGDMAPE } \\
=20.39 \%\end{array}$ & $\begin{array}{l}\text { MGDMAPE = Multivariate Gradient } \\
\text { Descent Mean Absolute Percentage } \\
\text { Error }\end{array}$ \\
\hline 2013 & Kumar et al & $\begin{array}{l}\text { Traffic flow and } \\
\text { speed(15-minute) }\end{array}$ & $\begin{array}{l}\text { Artificial Neural } \\
\text { Network (ANN) }\end{array}$ & $\mathrm{MAE}=37 \%$ & MAE = Mean Absolute Error \\
\hline 2014 & $\begin{array}{l}\text { Jian Wang, } \\
\text { Deng, \& } \\
\text { Guo }\end{array}$ & $\begin{array}{l}\text { Traffic flow (15- } \\
\text { minute) }\end{array}$ & $\begin{array}{l}\text { Advanced Bayesian } \\
\text { combination method }\end{array}$ & $\mathrm{MAPE}=13.2 \%$ & Mean Absolute Percentage Error \\
\hline \multirow[t]{2}{*}{2015} & \multirow[t]{2}{*}{ J. tang et al. } & \multirow[t]{2}{*}{ Traffic flow } & \multirow{2}{*}{$\begin{array}{l}\text { Genetically designed } \\
\text { hybrid model }\end{array}$} & $\operatorname{MDAPE}(\%)=14.19$ & Median Absolute Percentage Error \\
\hline & & & & $\begin{array}{l}\text { Average RMSE = } \\
42.84 \%\end{array}$ & RMSE = Root Mean square Error \\
\hline 2016 & Cai et al. & $\begin{array}{l}\text { Traffic flow and } \\
\text { speed (Hourly) }\end{array}$ & $\begin{array}{l}\text { Spatiotemporal } \\
\text { correlative k-nearest } \\
\text { neighbor model }\end{array}$ & $\operatorname{MAPE}(\%)=15.99$ & $\begin{array}{l}\text { Mean Absolute Percentage } \\
\text { Error(MAPE) }\end{array}$ \\
\hline
\end{tabular}


Table 1 shows the short summary of previous studies, data types and forecasting techniques

191 used. The limitations of the existing research lie in that most of the models carry out short-term

192 traffic forecasting at the link level, using univariate variable (historical traffic flow) and with an

193 unsatisfactory accuracy.

\subsection{Introduction to GA}

Researchers have applied GAs to a wide range of difficult optimization problems that are

197 quite challenging to solve using traditional mathematical models. Mathematical models use 198 mathematical techniques to solve the problem. These models are used to solve a variety of 199 optimization problems related to transportation planning, designing, and scheduling but it is quite 200 challenging to solve using traditional mathematical models. The advantage of mathematical 201 models is that mostly high-level management understands them easily as these models provide 202 definite solution. On the other hand, they have a few drawbacks as well. In traditional mathematical 203 models, it is quite hard to specify mathematical relationships analytically. Regarding these issues, 204 the genetic algorithm can provide an alternative way when the mathematical model becomes 205 inefficient due to high execution time in solving difficult problems (e.g., selecting a set of final 206 input variables from a large number of candidate input variables). Genetic algorithm is a heuristic 207 method, which is used to find the global minimum in the search domain, help to determine which 208 individuals should survive, which should reproduce, which should no longer survive and should 209 die. The mathematical form of GA was first developed by John Holland 1975. It is one of the most

210 widely used stochastic search methods to solve various problems and its solutions are usually far 211 better than other random search methods (Davis \& Nihan 1991). The GAs usually breeds according 212 to a predetermined, fixed number of generations and further these generations are populated with 
213 predetermined fixed number of binary strings. The main advantages of GAs over other search

214 methods are that GAs can solve problems using "the string" fitness to direct the search. GAs

215 require no specific knowledge regarding the search space and it works on population rather than

216 an individual point in the search space, which makes this algorithm more attractive than other

217 gradient search methods. The GAs need only a single measure of flow just to compare an

218 individual to the other individual. The performance of any genetic algorithm generally depends on

219 the type of operators used. The main operators of the genetic algorithm are crossover, mutation, 220 fitness and selection.

221 In the genetic algorithm, crossover is the procedure of picking out two members of the 222 population for the reproduction process. The operation of mutation allows a new individual to be 223 created. It begins by selecting an individual from the population based on its fitness. Fitness is 224 probably the main concept in Darwinian evolutionary theory. Fitness is measured in terms of an 225 individual's ability to compete for the resources to survive. The selection process refers as 226 choosing one or two parents from the population of individuals for the reproduction process. The 227 selection process is based on an individual's fitness value, higher the fitness value, more chances 228 to be selected to continue the reproduction process and eventually it will increase the convergence 229 of the genetic algorithm. The process of selecting an individual is one of the major aspects of 230 tuning the GA algorithm.

\section{3. Data Format and Initial Processing}

233 In this study, data from four urban arterial lanes are used to scrutinize traffic flow, speed

234 and occupancy patterns during peak and off-peak hours of each weekday. The data are collected

235 from an urban arterial road in Beijing, China using loop detectors in intervals of 5-minute. The 
236 input data set contains 3 months (July to September) traffic flow, speed and occupancy

237 information. The data are further filtered by deleting the weekends. Normally, weekends are the

238 least congested with smoother traffic during peak and off-peak hours. Data extraction tool was

239 designed to extract data in 5-min intervals (288 observations each weekday) for both peak and off-

240 peak periods.

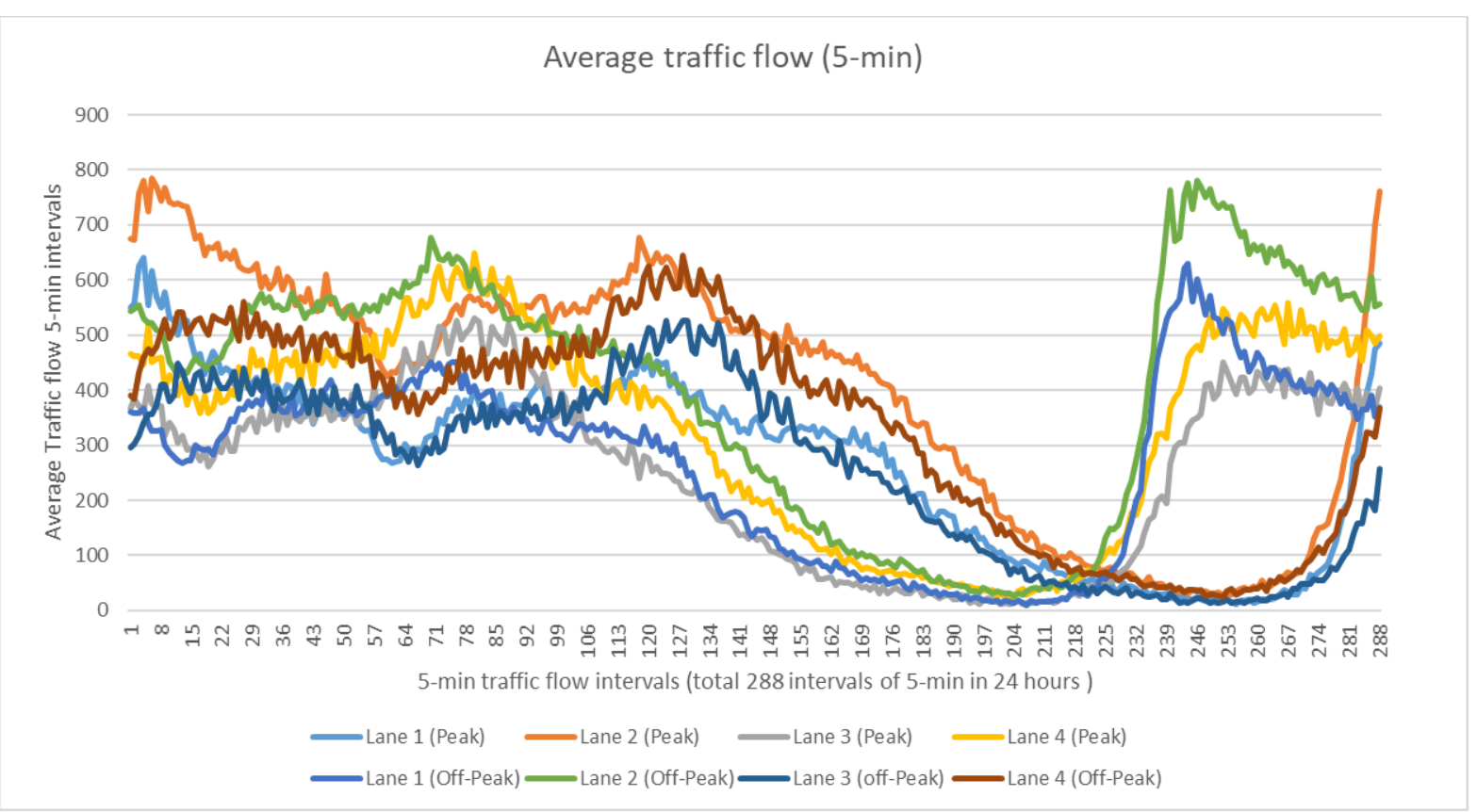

Fig. 1 Average peak and off-peak traffic flow (5-min intervals)

Fig 1 shows average peak and off-peak traffic flow only in intervals of 5-min. Fig 1 shows models are developed so that a single model is used to forecast traffic parameters (flow, density or speed) of six observations of 5-min each during weekdays (e.g., Peak time: ob1: 7:25 am to 7:30 am, ob2: 7:30 am to 7: 35 am, ob3: 7:35 am to 7:40 am, ob4: 7:40 am to 7:45 am, ob5: 7:45 am to 250 7:50 am, ob6: 7:50 am to $7: 55 \mathrm{am})$. Meanwhile, the peak and off-peak period, the disaggregate 251 models are developed so that one model is developed for forecasting a particular traffic parameter 
252 during a given 5-min interval over weekdays (peak: 7:25 am to 7:30 am; off-peak 11:25 am to $253 \quad 11: 30 \mathrm{am})$.

Fig. 2 Data collection and extraction flowchart

257 Fig 2 shows data collection and extraction flowchart for the study models. Both peak and off-peak 258 time traffic flow speed and occupancy data sets are used to train and test the proposed models.

\section{$260 \quad 3.1$ Study models}

261 Although various methodologies have been proposed and applied for, forecasting short-term urban

262 traffic, and the ultimate goal remains the same: to obtain the forecasting results as precisely and 263 quickly as possible. Researchers have been focusing on developing such methodologies, which are 264 used to improve and optimize the existing methods to provide better short-term traffic forecasting 265 tools. 


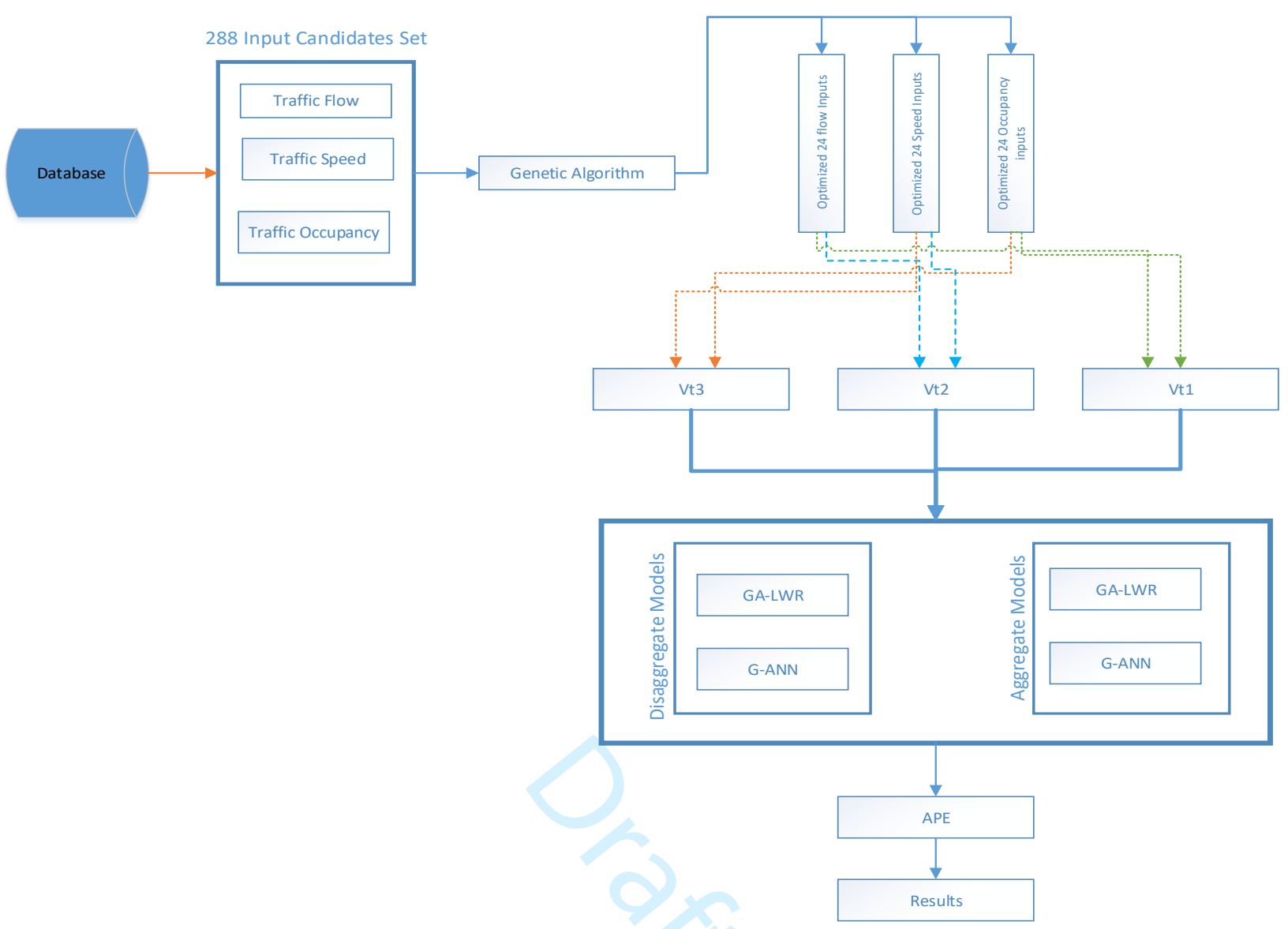

The data were extracted separately for traffic flow, speed and occupancy (Fig. 3). Later, 269 genetic algorithm is designed to optimize the extracted data sets. Within each generation, the 270 objective function of the genetic algorithm is to extract those independent variables from a set of 271 candidate variables, which have the highest linear correlation with the dependent variable by 272 assuming that this period contains all the necessary information needed to predict next 5-min 273 traffic flow, speed and occupancy. Then GA algorithm will evolve and produce the next generation, and the same objective 275 function is applied to pick up the subset, which has the highest linear correlation with the 
276 dependent variable. This process repeats until either the number of generations to be evolved is 277 reached or GA is converged.

278 Finally, genetically designed artificial neural networks (ANN) and locally weighted 279 regression (LWR) multivariate models are developed to forecast lane-based (5-min) traffic 280 variables such as;



$-\mathrm{V}_{\mathrm{t}}=$ Traffic flow at time $\mathrm{t}$ the case there are missing values within one or more input variables (Equation 1,2 and 3).

\section{Traffic Prediction Using GA Designed ANN and LWR Multivariate Models}

292 The fitness function evaluates how good a solution is. The fitness function makes sure that the best 293 individuals will continue to survive in the next generation and worst individuals will no longer 294 take part in the selection process and die. This process continues until the genetic algorithm reaches 295 the predefined termination criteria. The objective function of GA is designed to pick variables with 296 higher linear correlation. In the objective function of the genetic algorithm, the input data sets are 
297 converted into the regression matrix. The sum of square error (SSE) and R-squared are used to

298 find the relation between the independent variables and dependent variables. The R-squared values

299 are assigned to all input candidates. These objective scores are further converted into fitness scores

300 by comparing every individual candidate against another individual candidate in the population.

301 The mathematical formula for the objective function used to compute the linear correlation

302 coefficient " $r$ " is given as:

303

$$
r=\frac{n \sum x y-\left(\sum x\right)\left(\sum y\right)^{304}}{\sqrt{n\left(\sum x^{2}\right)-\left(\sum x\right)^{2}} \sqrt{n\left(\sum y^{2}\right)-\left(\sum y\right)^{2}}}
$$

Where, " $r$ " is called as linear correlation coefficient which is used to find the linear

308 relationship between two sets of variables (independent and dependent variables), " $\mathrm{n}$ " represents

309 the number of pairs of the data being used. Normally, the value of $\mathrm{r}$ resides between $-1<\mathrm{r}<$

$310+1$. The "+" sign represents the positive linear relationship while "_- "represents the negative linear

311 relationship among the variables. If " $\mathrm{r}$ " is greater than 0.8 , it means both variables have strong

312 linear relationship and if " $r$ " is less than 0.5 it means both variables have weak linear relationship

313 while 0 means there is no linear correlation among the variables or have a very weak relationship.

314 Finally, the designed genetic algorithm is used to optimize the given input data sets (flow,

315 speed and occupancy). The GA selects a set of final variables from 288 candidate variables 316 (independent variables) of higher linear correlation. 




318 Fig. 4 The relationship between the fitness value of GA objective function and number of final input variables for traffic parameters.

It is evident from the fitness values that set of 24 final variables show higher r-squared 322 value (Fig. 4) for all four lanes. Hence, the set of 24 candidates from 288 candidates are selected 323 as input to GA-ANN and GA-LWR models.

\subsection{Traffic prediction using GA designed multivariate ANN model}

327 The final input variables from GA are divide into two samples. The training sample is use to train 328 the Artificial Neural Network, while the testing sample is use to track training errors, which will 329 help to prevent overtraining problems. For GA-ANN aggregate multivariate model, 70\% (20 days: $33020 \times 6=120$ observations) is used for training purpose while $30 \%$ ( 9 days: $9 \times 6=54$ observations) 331 is used for testing purpose. Meanwhile, for GA-ANN disaggregate multivariate model; 70\% (20 332 days: $20 \times 1=20$ observations) is used for training purpose while remaining $30 \%$ ( 9 days: $9 \times 1=9$ 333 observations) is used for testing purpose. 
335 12. Empirical rule states that the number of hidden neurons in ANN should be equal to half or less

336 than the total inputs (Panchal et al. 2011). Trial and error approach has been used to build best

337 performance network. The final GA-ANN structure in the study contains 24 inputs, 12 neurons in

338 hidden layer and single output.

$340 \quad 4.2$ The prototype of ANN and LWR aggregate and disaggregate multivariate models

$341 \quad$ For peak and off-peak time traffic parameters prediction, the GA designed ANN (GA-

342 ANN) and LWR (GA-LWR) aggregate multivariate models contain six observations of 5-min

343 (Flow, speed and occupancy) each weekday. The GA-ANN and GA-LWR aggregate multivariate

344 models are used to predict peak and off-peak traffic parameters for 30-min window. However, the

345 GA designed ANN and LWR disaggregate multivariate models contain one observation of 5-min

346 window for both peak and off-peak. 


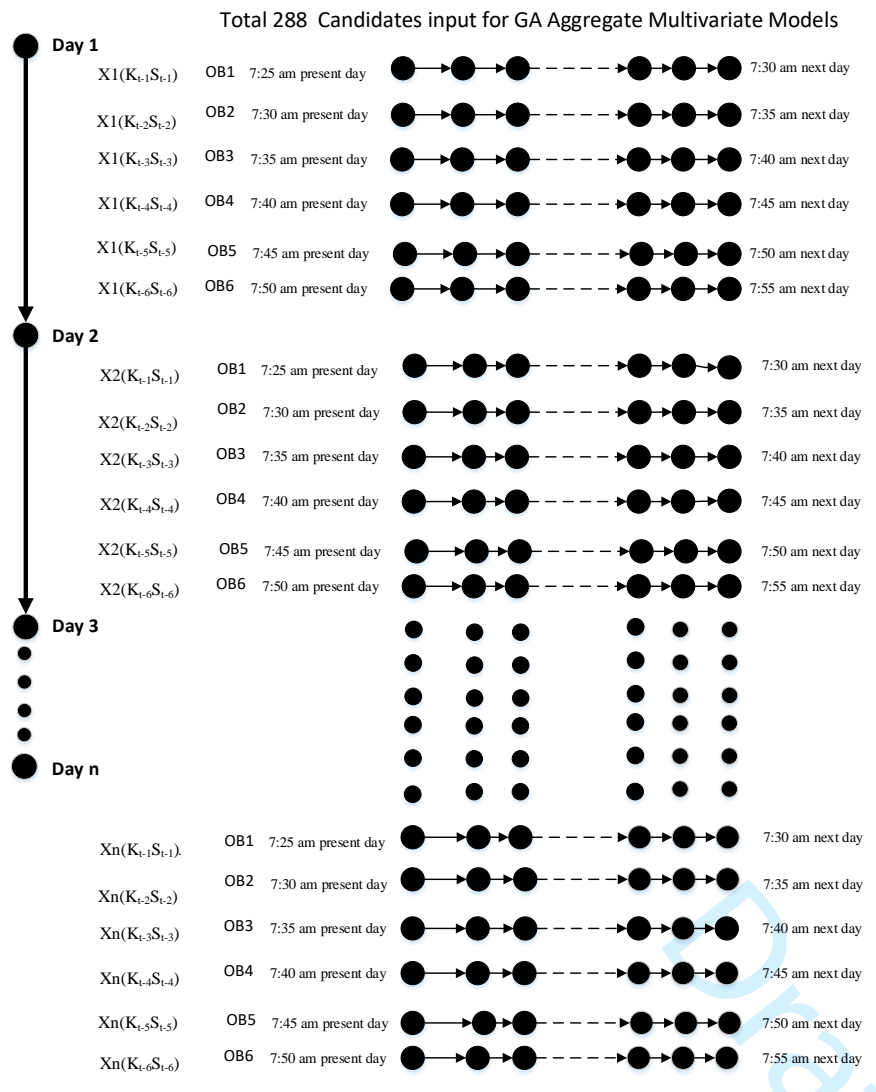

288 Candidates input for GA Disaggregate Multivariate Models
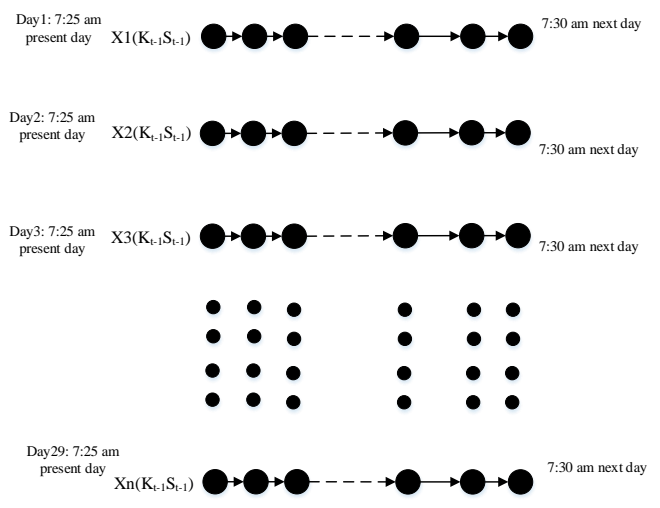

$353-$ First observation = I

$354-$ Last observation $(\mathrm{OB} 6)=\mathrm{I}+5$

355 The variables for developing ANN and LWR disaggregate multivariate model are:

$356-\quad$ Total number of days $=\mathrm{n}(29)$ 
$-\mathrm{X} 1\left(\mathrm{~K}_{\mathrm{t}-1} \mathrm{~S}_{\mathrm{t}-1}\right)=$ first day $($ day $)$

358

$-\mathrm{Xn}\left(\mathrm{K}_{\mathrm{t}-1} \mathrm{~S}_{\mathrm{t}-1}\right)=\mathrm{n}$ days

To evaluate the prediction performance of GA-ANN and GA-LWR aggregate and

360 disaggregate multivariate models, Absolute Percent Error (APE), Mean Absolute Deviation

361 (MAD), Mean Square Error (MSE) Mean Absolute Percentage Error (MAPE) and Root-Mean-

362 Square Error (RMSE) is used.

\section{5. Study Results and Discussions}

364 The designed GA-ANN and GA-LWR aggregate and disaggregate multivariate models are used 365 to predict 5-min and 30-min traffic variables into future for each weekday. The prototype of ANN 366 and LWR aggregate and disaggregate multivariate models (for both peak and off-peak period) are 367 presented above in Fig.5. The aggregate and disaggregate multivariate models are designed and 368 tested for combination of multi traffic variables such as; $\mathrm{Vt}_{1}$ (occupancy and speed), $\mathrm{Vt}_{2}$ (volume 369 and speed) and $\mathrm{Vt}_{3}$ (volume and occupancy), during peak and off-peak periods. 
370 Table 2 The GA-ANN and GA-LWR disaggregate multivariate models for $\mathrm{V}_{\mathrm{t} 1}, \mathrm{~V}_{\mathrm{t} 2}$ and $\mathrm{V}_{\mathrm{t} 3}$ prediction (Lane 1)

\begin{tabular}{|c|c|c|c|c|c|c|c|c|c|c|c|c|}
\hline & \multicolumn{6}{|c|}{ GA-ANN } & \multicolumn{6}{|c|}{ GA-LWR } \\
\hline & \multicolumn{6}{|c|}{ Lane 1} & \multicolumn{6}{|c|}{ Lane 1} \\
\hline & \multicolumn{3}{|c|}{ Peak } & \multicolumn{3}{|c|}{ Off-peak } & \multicolumn{3}{|c|}{ Peak } & \multicolumn{3}{|c|}{ Off-peak } \\
\hline & $\mathrm{Vt}_{1}$ & $\mathrm{Vt}_{2}$ & $\mathrm{Vt}_{3}$ & $\mathrm{Vt}_{1}$ & $\mathrm{Vt}_{2}$ & $\mathrm{Vt}_{3}$ & $\mathrm{Vt}_{1}$ & $\mathrm{Vt}_{2}$ & $\mathrm{Vt}_{3}$ & $\mathrm{Vt}_{1}$ & $\mathrm{Vt}_{2}$ & $\mathrm{Vt}_{3}$ \\
\hline Average & $11.6 \%$ & $12.1 \%$ & $7.5 \%$ & $4.0 \%$ & $3.9 \%$ & $4.3 \%$ & $10.9 \%$ & $7.0 \%$ & $7.1 \%$ & $6.1 \%$ & $5.1 \%$ & $5.6 \%$ \\
\hline $9^{\text {th }}$ Percentile & $29.6 \%$ & $21.1 \%$ & $22.3 \%$ & $12.0 \%$ & $11.2 \%$ & $12.5 \%$ & $26.2 \%$ & $12.5 \%$ & $15.5 \%$ & $10.1 \%$ & $10.2 \%$ & $11.9 \%$ \\
\hline MAD & 0.26 & 0.59 & 0.10 & 0.05 & 1.44 & 1.52 & 0.27 & 1.58 & 1.86 & 0.22 & 0.72 & 0.92 \\
\hline MSE & 0.48 & 1.13 & 1.84 & 0.02 & 2.78 & 3.21 & 0.11 & 1.63 & 1.02 & 0.08 & 1.24 & 1.48 \\
\hline RMSE & 0.69 & 1.06 & 3.98 & 0.14 & 1.66 & 1.79 & 0.34 & 1.25 & 1.00 & 0.28 & 1.11 & 1.21 \\
\hline МАPE & 5.60 & 1.89 & 6.00 & 2.17 & 1.30 & 6.72 & 10.34 & 0.90 & 4.14 & 10.02 & 0.59 & 4.12 \\
\hline
\end{tabular}

Table 3 The GA-ANN and GA-LWR disaggregate multivariate models for $\mathrm{V}_{\mathrm{t} 1}, \mathrm{~V}_{\mathrm{t} 2}$ and $\mathrm{V}_{\mathrm{t} 3}$ prediction (Lane 4)

\begin{tabular}{|l|l|l|l|l|l|l|l|l|l|l|l|l|l|l|}
\hline & \multicolumn{9}{|c}{ GA-ANN } & \multicolumn{3}{c|}{ GA-LWR } \\
\hline & \multicolumn{3}{|c|}{ Lane 4 } \\
\hline & \multicolumn{3}{|c|}{ Peak } & \multicolumn{3}{c|}{ Off-peak } & \multicolumn{3}{c|}{ Lane 4 } \\
\hline & $\mathrm{Vt}_{1}$ & $\mathrm{Vt}_{2}$ & $\mathrm{Vt}_{3}$ & $\mathrm{Vt}_{1}$ & $\mathrm{Vt}_{2}$ & $\mathrm{Vt}_{3}$ & $\mathrm{Vt}_{1}$ & $\mathrm{Vt}_{2}$ & $\mathrm{Vt}_{3}$ & $\mathrm{Vt}_{1}$ & $\mathrm{Vt}_{2}$ & $\mathrm{Vt}_{3}$ \\
\hline Average & $21.4 \%$ & $9.5 \%$ & $16.6 \%$ & $5.6 \%$ & $4.6 \%$ & $5.5 \%$ & $15.6 \%$ & $7.6 \%$ & $22.3 \%$ & $7.5 \%$ & $3.3 \%$ & $7.8 \%$ \\
\hline 95 th & $42.7 \%$ & $28.9 \%$ & $36.8 \%$ & $11.7 \%$ & $9.2 \%$ & $12.9 \%$ & $37.9 \%$ & $17.6 \%$ & $46.3 \%$ & $12.9 \%$ & $9.7 \%$ & $14.7 \%$ \\
\hline MAD & 0.09 & 1.84 & 0.74 & 0.25 & 1.53 & 1.05 & 0.07 & 3.18 & 1.38 & 0.16 & 1.20 & 0.79 \\
\hline MSE & 0.03 & 1.73 & 0.86 & 0.13 & 1.98 & 1.54 & 0.01 & 1.02 & 1.17 & 0.04 & 3.98 & 1.47 \\
\hline RMSE & 0.17 & 1.31 & 0.93 & 0.36 & 1.40 & 1.24 & 0.09 & 1.00 & 1.08 & 0.21 & 2.00 & 1.21 \\
\hline MAPE & 7.26 & 1.46 & 8.32 & 6.53 & 1.05 & 3.42 & 12.61 & 2.53 & 14.56 & 7.20 & 0.79 & 2.38 \\
\hline
\end{tabular}


Table 2 shows peak and Table 3 shows off-peak time traffic prediction errors for GA-ANN and GA-LWR disaggregate multivariate models, which are found to provide most accurate results.

378 Please note that, due to the limited space, the results for all other models are not presented here. 379 For peak time traffic prediction, the GA-ANN aggregate multivariate models result in most of 380 average errors of 16-119\% and most of the 95th percentile errors of 45-348\%. Meanwhile, for off381 peak time traffic prediction, the GA-ANN aggregate multivariate models result in most of average 382 errors of $11-54 \%$ and most of the 95 th percentile errors of $28-285 \%$.

On the contrary, for peak time traffic prediction, the GA-LWR aggregate multivariate models result in most of average errors of $17-113 \%$ and most of the 95th percentile errors of 55385 503\%. Meanwhile, for off-peak time traffic prediction, the GA-LWR aggregate multivariate models result in most of average errors of $10-61 \%$ and most of the 95th percentile errors of 26$387312 \%$.

However, for off-peak traffic prediction, the GA-ANN disaggregate $\mathrm{Vt}_{1}$ multivariate model results in most of average errors of $4-5 \%$ and most of the 95 th percentile errors of $11-14 \%$ 390 for each of the four lanes. Meanwhile, for off-peak traffic prediction, the GA-ANN disaggregate $391 \mathrm{Vt}_{2}$ multivariate model results in most of average errors of 2-5\% and most of the 95th percentile 392 errors of 9-11\% for each of the four lanes. However, for off-peak traffic prediction, the GA-ANN 393 disaggregate $\mathrm{Vt}_{3}$ multivariate model results in most of average errors of 3-5\% and most of the 95th 394 percentile errors of $11-12 \%$ for each of the four lanes. 
397 lower than $10 \%$ for each of the four lanes. Meanwhile, for off-peak time prediction, the GA-LWR 398 disaggregate $\mathrm{Vt}_{1}$ and $\mathrm{Vt}_{3}$ multivariate models show most of average of 5-7\% and the $95^{\text {th }}$ percentile 399 errors of 10-15\%, as shown in Table 2 and Table 3.

For lane-based short-term traffic prediction, the GA-ANN and GA-LWR disaggregate 401 multivariate models show lower average and the 95th percentile errors. The developed GA-ANN 402 and GA-LWR Vt 2 multivariate model performs better, when compared to $\mathrm{Vt}_{1}$ and $\mathrm{Vt}_{3}$ multivariate 403 models. In complex designed models with multi variables (GA, ANN, and LWR) often need to 404 find best optimal designed configured to obtain best results. However, the developed models are 405 complicated due to its hybrid nature but the accuracy of the models compared to previous studies 406 showed promising results and they can be a powerful tool especially in traffic management and 407 control.

\section{6. Conclusions and Recommendations}

410 In this study, genetically designed ANN (GA-ANN) and LWR (GA-LWR) multivariate models

411 for forecasting short-term urban traffic are proposed and tested. Genetic algorithms are used to 412 optimize the input datasets. The input datasets are in intervals of 5-min window each weekday.

413 GA is used to optimally select 24 final input variables from the given candidate input dataset of 414288 initial input variables. The 24 final GA optimized datasets are input to ANN and LWR 415 multivariate models for prediction of traffic parameters.

In this study, two types of models are developed and tested. 1) Aggregate multivariate

417 model and 2) disaggregate multivariate model. Both models and their prototypes are already 418 discussed in earlier sections. The aggregate multivariate model is developed using traffic 
419 parameters data of every 5-min window with a 30-min window (e.g., 7:25 - 7:55 am peak and 420 11:25-11:55 am off-peak period from Monday to Friday). Meanwhile, disaggregate multivariate 421 model is developed based on data of a particular 5-min window of each weekday (7:25 - 7:30 am 422 for peak period and 11:25-11:30 am for off-peak period from Monday to Friday). The GA-ANN 423 and GA-LWR aggregate and disaggregate multivariate models are used to predict short-term 424 traffic parameters for 5-min and 30-min window respectively. It is found that, during the peak and 425 off-peak window, all four lanes show distinctive traffic patterns.

The proposed GA-ANN and GA-LWR disaggregate multivariate models show lower 427 forecasting errors and such robust models will predict traffic parameters in a highly accurate 428 manner. Short-term prediction of traffic parameters (e.g., such as flow, speed and density) is a 429 crucial element of current ITS, yet complex to accomplish mathematically because of their 430 dynamic nature. To accommodate the increasing traffic demand, better forecasting techniques 431 should be taken into account. In general, lane-based traffic forecasting with a temporal resolution 432 of 5-min window has rarely been studied before, especially with a combined input consisting of 433 multiple traffic parameters. The present study eliminates this existing research gap with the 434 proposed hybrid multivariate models coupling GA, ANN and LWR. The present study proposed 435 an optimized framework for modelling the dynamic of traffic through the forms of hybrid 436 multivariate neural network and locally weighted regression methods. multivariate models are used to predict lane-based short-term traffic parameters. To predict peak 439 and off-peak time 5-min traffic condition into future, the GA-ANN and GA-LWR models are 440 developed and tested. For peak and off-peak time traffic prediction, the GA-ANN and GA-LWR 
441 aggregate multivariate models show higher average errors and the 95th percentile errors. On the 442 other hand, for the peak and off-peak time traffic prediction, the GA-ANN and GA-LWR 443 disaggregate multivariate models show satisfactory results with lower average and 95th percentile 444 errors. Hence, for short-term traffic parameters prediction, the GA-ANN and GA-LWR 445 disaggregate multivariate models show lower average and 95th errors, when compared to GA446 ANN and GA-LWR aggregate multivariate models.

When compared to the previous studies, where most of the average errors are higher than

$44810 \%$ (Table 1), the genetically designed GA-ANN and GA-LWR disaggregate multivariate models 449 show lower average errors. Moreover, the GA-ANN and GA-LWR disaggregate multivariate 450 model $\mathrm{Vt}_{2}$ shows promising results where most of the average errors are lower than $5 \%$ for each 451 of four lanes. Nevertheless, for peak and off-peak time prediction, both GA-ANN and GA-LWR 452 models showed lower MSE of 0.11-1.84 when compared to previous studies (Table 1). Results 453 imply that genetically designed models have a much better ability to capture high traffic variation, 454 which is of primary importance to traffic prediction and major shortcoming of other approaches 455 such as ARIMA models. The contributions of this paper is to develop lane-based short-term 456 forecasting models using advanced techniques (such as GA, ANN, and LWR), which also have a 457 higher accuracy than the existing models reported in the literature.

A great care was taken to present plausible models for short-term traffic forecast. Despite 459 of this all, the present study has few limitations. Data were from a specific city and specific road 460 type; thus, models are only tested on data sets collected from that particular type of urban road. 461 Various factors such as road conditions, accidents, weather conditions, etc. are excluded during 462 the model design and development. A few related research topics can be explored in the future 
463 including that, the model should be tested on other facility types, such as freeways and rural road

464 types. Other parameters like weather condition and other information (e.g., accidents) should be 465 included to make models more robust.

\section{Acknowledgements}

467 The authors would like to acknowledge the funding from Hubei Provincial Natural Science 468 Foundation (2015CFB599) and the funding for Top 1\% ESI Academic Program from Wuhan 469 University of Technology supported by "the Fundamental Research Funds for the Central 470 Universities" (WUT:2014-VII-036). This study is also supported by a start-up grant from Wuhan 471 University of Technology.

\section{References}

474 Atkeson, C. G., Moore, A. W., and Schaal, S. 1997b. "Locally weighted learning for control." $475 \quad$ Artificial Intelligence Review. 11, 75-113.

476 Atkeson, C., Moore, A., \& Schaal. 1997. "Locally weighted learning.” AI Review., 11, 11-73.

477 Box, G., and Jenkins, J. 1970. “Time series analysis.” Forecasting and control, Holden-Day, San $478 \quad$ Francisco.

479 Centiner, B. G., Sari, M., \& Borat, O. 2010. "A neural network based traffic-flow prediction 480 model.” Mathematical and Computational Applications., 15, 269-278.

481 Cleveland, W., Devlin, S., Grosse, E. 1988. "Regression by local fitting.” Journal of 

Econometrics., 37, 87-114.

483 Davis, G.A., Nihan, N.L. 1991. "Nonparametric regression and short-term freeway traffic 484

Dougherty, M. S., and Cobbett, M. R. 1997. "Short-term inter-urban traffic forecasts using predicting." Journal of Transportation Engineering., 117(2), 178-188.

Friedman, J. H. 1995. “Intelligent local learning for prediction in high dimensions.” Proc., Int. neural networks.” Int. J. Forecast., 13, 21-31.

Hamed, M.M., Al-Masaeid, H.R., Bani Said, Z.M. 1995. “Short-term prediction of traffic volume in urban arterials.” ASCE Journal of Transportation Engineering., 121(3), 249254.

Holland, J. H. 1975. “Adaptation in natural and artificial systems.” University of Michigan Press, 493 Ann Arbor, Mich.

Hu, W., Liu, Y., Li, L., \& Xin, S. 2010. “The short-term traffic flow prediction based on neural network." 2nd International Conference on Future Computer and Communication., 1, 293-296. traffic flow forecasting". Transportation Research Part C: Emerging Technologies., 43(1), 79-94. 

forecasting." Journal of Transportation Engineering., 131, 771-779.

502

503

504

505

506

507

508

509

510

511

512

513

514

515

516

517

Khan, S.I. and P. Maini. 2000. "Modelling Heterogeneous Traffic Flow”. Transportation Research Record., 1678, 234- 241.

Kirby, H. R., Watson, S. M., \& Dougherty, M.S. 1997. "Should we use neural networks or statistical models for short-term motorway traffic forecasting.” International Journal of Forecasting., 13, 43-50.

Kumar, K., Parida, M., \& Katiyar, V. 2013. "Short Term Traffic Flow Prediction for a NonUrban Highway Using Artificial Neural Network.” Procedia - Social and Behavioral Sciences., 104, 755-764.

Lam, W.H.K., Tang, Y.F., Tam, M.-L. 2006. “Comparison of two non-parametric models for daily traffic forecasting in Hong Kong.” Journal of Forecasting., 25(3), 173-192.

Ledoux, C. 1997. “An urban traffic flow model integrating neural networks.” Transportation Research Part C., 5, 287-300.

Levin, M., Tsao, Y.-D. 1980. On forecasting freeway occupancies and volumes. Transportation Research Record., 773, 47-49.

Lingras, P., \& Adamo, M. 1996. "Average and Peak Traffic Volumes: Neural Nets, Regression, Factor Approaches." Journal of Computing in Civil Engineering., 10(4), 300-306.

Liu, Y., Shao, X., \& Li, X. 2007. "Short-term traffic flow prediction model based on Lagrange support vector regression." Journal of transportation information and safety., 29(4), 46- 
Mei, H., Ma, A., Poslad, S., \& Oshin, T. O. 2015. "Short-Term Traffic Volume Prediction for Sustainable Transportation in an Urban Area.” Journal of Computing in Civil Engineering., 29(2), 04014036.

Okutani, I., Stephanedes, Y.J. 1984. “Dynamic Prediction of Traffic Volume Through Kalman Filtering Theory." Transportation Research, Part B., 18(1), 1-11.

Pamula, T. 2011. "Road traffic parameters prediction in urban traffic management systems using neural networks." Transport Problems., 6, 23-28.

Panchal, Gaurang., Ganatra, Amit., Kosta, Y. P. 2011. "Panchal, Devyani. Behaviour analysis of multilayer perceptrons with multiple hidden neurons and hidden layers.” In IJCTE., 332337.

Pinlong Cai, Yunpeng Wang, Guangquan Lu, Peng Chen, Chuan Ding, Jianping Sun. 2016."A spatiotemporal correlative k-nearest neighbor model for short-term traffic multistep forecasting”. Transportation Research Part C: Emerging Technologies.,62, 21-34.

Quek, C., Pasquier, M., Lim, B.B.S. 2006. “POP-TRAFFIC: a novel fuzzy neural approach to road traffic analysis and prediction.” IEEE Transactions on Intelligent Transportation Systems., 7(2), 133-146. Approach.” Transportation Research Board., 1453, 98-104. 
Smith, B.L., Demetsky, M.J. 1997. “Traffic Flow Forecasting: Comparison of Modelling Approaches.” Journal of Transportation Engineering., 123(4), 261-266.

Smith, B.L., Williams, B.M., Oswald, K.R. 2002. "Comparison of parametric and nonparametric models for traffic flow forecasting." Transportation Research Part C: Emerging Technologies., 10(4), 303-321.

Sun, H., Liu, H.X., Xiao, H., He, R.R., Ran, B. 2003. "Short-term traffic forecasting using the local linear regression model.” Transportation Research Board 82nd Annual Meeting, Washington, DC.

Tang, J., Zhang, G., Wang, Y., Wang, H., Liu, F. 2015. “A hybrid approach to integrate fuzzy Cmeans imputation meth with genetic algorithm for missing traffic flow data estimation." Transportation Research Part C., 51, 29-40.

Theja, P. V. V. K., \& Vanajakshi, L. 2010. "Short Term Prediction of Traffic Parameters Using Support Vector Machines Technique.” Proceedings of third international conference on Emerging trends in Engineering and Technology., 70-75.

Turochy, R. 2006. "Enhancing short-term traffic forecasting with traffic condition information." Journal of Transportation Engineering., 132(6), 469-474.

Vlahogianni, E. I., Karlaftis, M. G., \& Golias, J. C. 2014. "Short-term traffic forecasting: Where we are and where we're going.” Transportation Research Part C: Emerging Technologies, 43(1), 3-19.

Vlahogianni, E.I., Karlaftis, M.G., Golias, J.C. 2005. “Optimized and meta-optimized neural 
networks for short-term traffic flow prediction: a genetic approach." Transportation Research Part C., 13(3), 211-234.

Wang, J., \& Shi, Q. 2013. "Short-term traffic speed forecasting hybrid model based on ChaosWavelet Analysis-Support Vector Machine theory." Transportation Research Part C: Emerging Technologies., 27, 219-232.

564 Williams, B.M., Durvasula, P.K., Brown, D.E. 1998. "Urban Traffic Flow Prediction: Application of Seasonal Autoregressive Integrated Moving Average and Exponential Smoothing Models.” Transportation Research Record., 1644, 132-144.

Xie, Y.C., Zhang, Y.L. 2006. “A wavelet network model for short-term traffic volume forecasting.” Journal of Intelligent Transportation Systems., 10(3), 141-150.

Yasdi, R. 1999. "Prediction of road traffic using a neural network approach.” Neural Computing and Applications., 8, 135-142.

Yonghui, S., Jun, B., Zhongzhen, Y., \& Shengping, J. 2011. "Hybrid intelligent model for urban road short -term traffic flow prediction.” Journal of transportation information and safety., 25(5), 58-61. ARIMA and ANN models.” Workshop on Power Electronics and Intelligent Transportation System., 621-625. genetic, neural, and regression techniques." Transportation Research Part C: Emerging 

Technologies., 12, 139-166.

580 Zhong, M., Sharma, S., \& Lingras, P. 2005. “Short-Term Traffic Prediction on Different Types 581 of Roads with Genetically Designed Regression and Time Delay Neural Network

582 Models.” Journal of Computing in Civil Engineering., 19(1), 94-103. 